\title{
Une intervention brève auprès de parents adoptants centrée sur la sensibilité parentale : effets sur le sentiment de compétence parentale et l'attachement de l'enfant
}

\author{
Brief intervention for adopted parents focusing on parental sensitivity: \\ Impact on parental self-efficacy beliefs and child's attachment \\ M.-N. De Theux-Heymans ${ }^{b}$, M. Stievenart ${ }^{\mathrm{a}, *}{ }$ I. Roskam $^{\mathrm{a}}$ \\ ${ }^{a}$ Université catholique de Louvain, institut de recherches en sciences psychologiques, 10, place du Cardinal Mercier, \\ 1348 Louvain-la-Neuve, Belgique \\ b a.s.b.l. L'Envol, 2 C, rue de France, 1470 Genappe, Belgique
}

Reçu le 11 octobre 2012 ; accepté le 17 avril 2013

\section{Résumé}

L'enfant adopté semble présenter pour lui-même et pour ses parents adoptifs des défis spécifiques en ce qui concerne la récupération de son attachement. Basée sur l'hypothèse du lien entre la sensibilité parentale des parents adoptant et l'attachement de l'enfant, cette étude vise à créer une intervention centrée sur la sensibilité parentale pour des familles adoptives belges francophones. Elle a été testée auprès de neuf familles adoptives dont les enfants avaient entre deux et 11 ans. Dans cette démarche exploratoire, la récupération de l'attachement et la modération du sentiment de compétence parentale ont été évaluées. Les tendances qui s'en dégagent mettent en évidence la pertinence de répliquer plus largement cette intervention.

(C) 2013 Société française de psychologie. Publié par Elsevier Masson SAS. Tous droits réservés.

Mots clés : Intervention ; Adoption ; Sentiment de compétence ; Attachement ; Sensibilité parentale

\section{Abstract}

Adopted children seem to imply specific challenge for themselves and their adoptive parents regarding the recovery of their attachment representations. Based on the hypothesis relating parental sensitivity of adopting parents and children's attachment representations, this study aims to create an intervention based

\footnotetext{
* Auteur correspondant.

Adresse e-mail : marie.stievenart@uclouvain.be (M. Stievenart).
} 
on parental sensitivity for adoptive French-speaking families. Nine adoptive families took par to this study whose children are between 2 and 11 years old. In this exploratory research, the recovery of the children's attachment representations and the moderation of parental self-efficacy were assessed. Our results highlighted the relevance of this intervention.

(C) 2013 Société française de psychologie. Published by Elsevier Masson SAS. All rights reserved.

Keywords: Intervention; Adoption; Self-efficacy beliefs; Attachment; Parental sensitivity

\section{Introduction}

Depuis une vingtaine d'années, de nombreux programmes anglophones de prévention et d'intervention, adressés aux parents, se développent visant à soutenir l'attachement de leur enfant. Parmi ceux-ci, quelques-uns visent spécifiquement les besoins des familles adoptives.

En Belgique francophone, le cadre légal prévoit que, durant la période précédant l'arrivée de l'enfant, les parents candidats à l'adoption participent à des ateliers et entretiens alors qu'ils ne sont pas tenus d'être accompagnés durant la période post-adoptive. Pourtant, les repères d'éducation «classiquement» répandus ne suffisent pas toujours à guider les parents adoptants de manière optimale dans la construction du nouveau lien avec l'enfant. Les professionnels soulignent la nécessité d'intervenir auprès de ceux-ci (Tyrrell \& Dozier, 1999; van den Dries, Juffer, van Ijzendoorn, \& Bakermans-Kranenburg, 2009). Dans ce contexte, nous avons tenté de développer une intervention à destination des parents adoptants afin qu'ils puissent utiliser les notions de base de la théorie de l'attachement avec leur enfant adopté, au cours des premières années dans sa nouvelle famille.

\subsection{Quelques notions de la théorie de l'attachement}

Dans le contexte de l'adoption, la théorie de l'attachement peut apporter un éclairage important sur les processus en jeu. Elle présente une clé de lecture des difficultés rencontrées par les familles adoptives.

Du point de vue théorique, l'enfant dispose d'un système d'attachement dont l'objectif est, en cas d'alarme, d'assurer sa survie et son réconfort via la proximité avec la figure d'attachement, bien souvent le parent (Bowlby, 1969/1982). L'enfant est également doté d'un système qui le pousse à découvrir son environnement, le système exploratoire, antagoniste au système d'attachement. Le premier s'active lorsque l'enfant se sent effrayé ou en détresse (le second étant alors désactivé) et s'éteint lorsqu'il se sent en sécurité (le second étant activé). Ces différents systèmes se mettent en place grâce à la base de sécurité, incarnée par la figure d'attachement et assurant deux fonctions : soutenir l'exploration de l'enfant et lui apporter réconfort et protection en cas de besoin (Ainsworth, Blehar, Waters, \& Wall, 1978).

Des différences individuelles quant à la qualité de l'attachement et de l'exploration apparaissent en fonction du système de caregiving du parent, référant à la capacité de celui-ci à procurer des soins adéquats en réponse aux besoins de l'enfant (George $\&$ Solomon, 2008). Dans la mesure où le parent prend soin de son enfant avec sensibilité, l'enfant développe une confiance en son parent comme étant accessible en cas de besoin. Dès lors, il peut explorer librement son environnement. Quand il est en détresse, il se tourne alors vers le parent pour être rassuré et apaisé. Il s'agit de l'attachement sécure (Ainsworth et al., 1978). À l'inverse, si le parent répond à la détresse 
de l'enfant en le rejetant ou de manière inconsistante et imprévisible, celui-ci développe un attachement insécure. Dans la mesure où le système d'attachement n'est pas désactivé suite aux réponses du parent, l'attachement insécure compromet l'exploration. Il est possible aussi que le parent adopte des comportements contradictoires, hostiles et sans aide pour la protection de l'enfant, ce dernier est alors face à un dilemme de "peur sans solution » : il se dirige vers le parent pour être rassuré alors que celui-ci l'effraie. L'enfant présente alors un attachement désorganisé.

Comme décrit ci-dessus, les parents interviennent dans l'élaboration d'un attachement sécure de leur enfant. En effet, des différences dans le système de caregiving du parent influencent l'attachement de l'enfant. Celles-ci peuvent trouver leur origine dans la sensibilité parentale définie comme la capacité du parent à percevoir les signaux d'attachement de son enfant avec acuité et à y répondre rapidement, de manière cohérente et adéquate (Ainsworth et al., 1978). Ainsi, les interventions orientées vers la sensibilité parentale cherchent à développer celle-ci en amenant le parent à percevoir correctement les signaux d'attachement de son enfant, et à y répondre rapidement et adéquatement (Bakermans-Kranenburg, van Ijzendoorn, \& Juffer, 2008).

\subsection{La sensibilité parentale: un préalable aux différences individuelles d'attachement}

L'intervention créée dans le cadre de cette recherche se base sur une hypothèse fondamentale de la théorie de l'attachement, à savoir l'influence de la sensibilité parentale sur le développement de l'attachement chez l'enfant. Dans une méta-analyse investiguant les antécédents parentaux de l'attachement de l'enfant, De Wolff et van Ijzendoorn (1997) concluent que la sensibilité parentale, principalement maternelle, en est un déterminant important.

Cette sensibilité parentale peut être influencée par le sentiment de compétence du parent, défini comme la perception par celui-ci de sa capacité à influencer positivement le comportement et le développement de son enfant (Jones \& Prinz, 2005). Ce sentiment de compétence peut influencer la manière dont le parent se comporte avec son enfant, et donc son système de caregiving incluant la sensibilité parentale (e.g. Coleman \& Karraker, 1998 ; Weinfield, Whaley, \& Egeland, 2004). Ainsi, il a été démontré que les parents d'enfants présentant un attachement désorganisé sont caractérisés par l'abdication de leur système de caregiving: ils se considèrent incapables de protéger leur enfant et se sentent impuissants (George \& Solomon, 2008). Ce sentiment de compétence bas engendre d'importantes conséquences négatives sur leur sensibilité parentale.

\subsection{En quoi est-il pertinent de créer une intervention renforçant l'attachement de l'enfant adopté?}

L'enfant adopté entre dans sa famille adoptive avec un bagage d'expériences ayant déjà façonné son attachement. Dans ces conditions, les enfants adoptés peuvent-ils développer un attachement sécure ? Les résultats ne sont pas convergents. Ainsi, la méta-analyse de van den Dries et al. (2009) met en lumière que les enfants adoptés montrent significativement moins d'attachement sécure que des enfants biologiques alors que d'autres études mettent en évidence que les enfants adoptés présentent un attachement sécure dans des proportions similaires aux enfants biologiques (Juffer \& Rosenboom, 1997 ; Ponciano, 2010). Ces différences dans les résultats peuvent s'expliquer par la prise en compte, ou pas, de certains facteurs, tels que l'âge de l'enfant lors de la séparation et les changements d'environnement, pouvant influencer l'attachement de l'enfant (Euillet, Spencer, Troupel-Cremel, Fresno, \& Zaouche-Gaudron, 2008).

En ce qui concerne la question de savoir si les enfants adoptés développent un attachement désorganisé, les recherches semblent assez unanimes: les enfants adoptés présentent plus 
fréquemment un attachement désorganisé que les enfants biologiques (e.g. O’Conner, Marvin, Rutter, Olrick, \& Britner, 2003 ; Ponciano, 2010 ; van Londen, Juffer, \& van Ijzendoorn, 2007). Dans leur parcours de vie, ces enfants ont souvent été confrontés à des situations de négligence et de maltraitance familiale ou institutionnelle, menant souvent à de la "peur sans solution», démontrée comme induisant de la désorganisation (e.g. Cyr, Euser, Bakermans-Kranenburg, \& Van Ijzendoorn, 2010; Juffer, Bakermans-Kranenburg, \& van Ijzendoorn, 2005 ; Marcovitch, Goldberg, Gold, \& Washington, 1997). C'est pourquoi la récupération de la désorganisation de l'attachement, même partielle, durant l'enfance est un objectif déterminant pour le développement de l'enfant.

Par ailleurs, les enfants adoptés peuvent développer un nouvel attachement avec le parent adoptant malgré l'attachement développé dans leur vie pré-adoptive (Howes \& Spieker, 2008). Cependant, durant la phase de construction de l'attachement au parent adoptant, les enfants adoptés sélectionnent dans leurs interactions avec ce dernier ce qui vient confirmer leur attachement préalable (Miljkovitch, Pierrehumbert, Karmaniola, \& Halfon, 2003). C'est pourquoi les interventions visant la récupération des dégâts de l'adversité précoce de l'histoire de vie pré-adoptive de l'enfant sont pertinentes (Dozier, Lindhiem, \& Ackerman, 2005). Il s'agit de soutenir la récupération de l'attachement grâce à une grande sensibilité de la part du parent adoptant, c'est-à-dire une vigilance à tenter de comprendre le vécu émotionnel, les comportements et les pensées de l'enfant sur base de son vécu pré-adoptif. Dans ce contexte, la parentalité adoptive nécessite des connaissances et compétences complémentaires : il ne s'agit pas d'être simplement un parent de substitution mais également de devenir un parent thérapeutique (Dozier et al., 2005).

\subsection{Les défis de la parentalité adoptive concernant l'attachement}

Pour être une base de sécurité pour l'enfant, le parent doit se montrer disponible et attentif aux signaux de celui-ci, et prêt à fournir de l'aide et du réconfort s'il perçoit que l'enfant en a besoin (e.g. Meins, Fernyhough, Fradley, \& Tuckey, 2001 ; Slade, 2005). Cependant, tout parent expérimente inévitablement des conflits entre le besoin de rester disponible et ses propres préoccupations. La recherche d'un équilibre entre les deux requiert une grande flexibilité (George \& Solomon, 2008). Cela est encore plus délicat pour un parent adoptant dans la mesure où l'absence de condition favorisant la transition à la parentalité peut gêner le plein épanouissement du système de caregiving. Dès lors, un prérequis à un caregiving adéquat du parent adoptant consiste à favoriser sa disponibilité émotionnelle en éveillant sa sensibilité aux comportements d'attachement spécifiques à son enfant adopté (Schofield \& Beek, 2006).

De plus, ayant souvent développé un attachement empreint de méfiance, les enfants adoptés peuvent avoir tendance à se comporter de manière rejetante à l'égard du parent (Stovall \& Dozier, 2000, 2004), ce qui peut avoir des répercussions négatives sur le système de caregiving de ce dernier. Le mécanisme d'ajustement entre le parent et l'enfant adopté semble jouer un rôle important durant les premières semaines dans la famille. Dans les dyades où l'enfant est insécure, le parent, malgré un attachement sécure, a tendance à répondre de manière complémentaire aux comportements de l'enfant. Par exemple, le parent ignore les comportements d'attachement, bien souvent dissimulés derrière une apparente indifférence, caractéristique de l'attachement insécure, de l'enfant. De manière étonnante, c'est l'enfant et non le parent qui «mène » l'interaction. Ces observations soulignent l'importance de soutenir l'apprentissage du parent adoptif à reconnaître les comportements adaptatifs et défensifs que l'enfant a développés avant d'arriver dans la famille adoptive. Il peut ainsi interpréter correctement les signaux de son enfant et y répondre 
avec sensibilité même quand l'enfant le repousse ou l'ignore (Dozier, Higley, Albus, \& Nutter, 2002).

\subsection{Interventions auprès des parents adoptants}

Définir les orientations principales de notre intervention nécessite d'analyser les interventions ayant déjà fait leurs preuves. Selon Bakermans-Kranenburg et al. (2008), les interventions brèves se focalisant strictement sur la sensibilité parentale sont les plus efficaces pour améliorer l'attachement de l'enfant.

Dans la littérature, nous avons répertorié quatre interventions standardisées s'adressant aux familles adoptives. «L'intervention relationnelle» (IR, pour une description voir Moss, DuboisComtois, Cyr, Carignan, \& St-André, 2009) s'adresse à des familles adoptives avec un enfant en âge préscolaire. Deux autres interventions, «Attachment and bio-behavioral catch-up » (ABC, pour une description voir Dozier et al., 2005) et « Video-feedback intervention to promote positive parenting » (VIPP, pour une description voir Juffer, Bakermans-Kranenburg, \& Van Ijzendoorn, 2008) s'adressent à des familles adoptives avec des enfants en bas âge. Une quatrième intervention, «Circle of security » (COS, pour une description voir Marvin, Cooper, Hoffman, \& Powell, 2002) a été prise en compte même si elle n'a pas été évaluée auprès de familles adoptives. Elle présente des supports originaux inspirés de la théorie de l'attachement et plusieurs caractéristiques similaires au contexte de notre intervention, telles que l'âge des enfants et des activités menées en groupe.

Les objectifs annoncés de ces quatre interventions reprennent, entre autres, la nécessité de procurer des soins sensibles à son enfant même lorsqu'il ne le demande pas. Au regard des différentes activités proposées par chaque programme pour soutenir la sensibilité parentale, il s'agit d'aider le parent à prendre conscience des besoins d'attachement de l'enfant sécure, à réinterpréter les signaux de l'enfant insécure, et à développer des capacités d'observation et de déduction.

En ce qui concerne les effets de ces interventions, ils peuvent être évalués soit chez les parents participant à l'intervention, soit chez les enfants bénéficiant des changements apportés par l'intervention (Berlin, 2005). L'évaluation de l'ABC démontre que les enfants présentent moins d'attachement désorganisé au terme de cette intervention et sont significativement plus enclins à développer un attachement sécure. Le VIPP a un effet significatif sur la sensibilité parentale et la sécurité d'attachement de l'enfant ainsi qu'une réduction de la désorganisation. Les mères ayant participé à l'IR ont une sensibilité plus élevée que celles du groupe témoin, sans que cela n'ait d'effet significatif sur la sécurité d'attachement de l'enfant. Cependant, les enfants dont la mère a participé à l'IR recherchent davantage la proximité avec celle-ci, ce qui indiquerait que l'intervention déclenche un processus de changement menant vers davantage de sécurité.

L'évaluation des trois interventions ci-dessus permet de dégager les conclusions suivantes. Une intervention brève (entre quatre et dix séances) centrée principalement sur la sensibilité parentale peut aboutir aux effets suivants :

- une légère amélioration de la sécurité d'attachement (ABC et IR);

- une diminution de la désorganisation (ABC et VIPP);

- une amélioration de la sensibilité parentale (VIPP et IR).

La diminution de la désorganisation est très prometteuse. En effet, comme présenté ci-dessus, l'adversité précoce vécue par les enfants adoptés induit des risques importants de présenter un 
attachement désorganisé (Dozier et al., 2005 ; Juffer et al., 2005 ; Ponciano, 2010 ; van den Dries et al., 2009). Deux pistes peuvent expliquer les résultats plus mitigés pour la sécurité :

- le changement de la sécurité pourrait être obtenu à long terme et donc ne peut être mesuré à l'issue de l'intervention;

- la sécurité serait déjà présente avant l'intervention, tel que suggéré par Juffer et Rosenboom (1997).

Le format et les activités de notre intervention peuvent être élaborés en fonction des objectifs définis par ces différents programmes. La progression de notre intervention sera basée sur celles de l'ABC et de l'IR car ces interventions sont créées à partir d'une étude minutieuse des besoins des familles adoptives et leurs objectifs sont identiques à ceux souhaités pour notre intervention. Toutefois, les activités proposées dans ces deux interventions ne peuvent être utilisées telles quelles car l'ABC et l'IR se réalisent en individuel à domicile et supposent la formation à des outils spécifiques dont nous ne disposons pas. C'est pourquoi nous nous sommes aussi inspirées d'outils et supports du COS, adressés à des enfants d'âge préscolaire.

\subsection{Développement de notre intervention}

Une analyse des interventions standardisées adressées aux parents adoptants montre que la réussite de ces programmes serait surtout due à : (1) l'utilisation de vidéo-feedback, (2) l'engagement des parents dans le programme et (3) la qualité de la relation entre parents et intervenants (Berlin, Zeanah, \& Lieberman, 2008). Ces repères ont fondé les choix du format, des principes méthodologiques et des supports exploités dans notre intervention.

Le vidéo-feedback (1) consiste à analyser finement les interactions entre le parent et son enfant à partir de séquences filmées. Durant le visionnement, l'intervenant met en avant les aspects positifs des interactions parent-enfant et aide le parent à repérer certains signaux de l'enfant afin d'y répondre adéquatement ultérieurement. Ces séances de vidéo-feedback améliorent les compétences d'observation du parent, influençant ainsi sa sensibilité parentale (Juffer et al., 2008). Cette méthode semble être incontournable pour les interventions centrées sur la sensibilité parentale. Toutefois, nous ne disposons pas de la formation nécessaire pour l'utiliser dans le cadre de notre intervention. Deux alternatives y pallient : l'utilisation de séquences vidéo d'autres dyades parent-enfant et le recours au journal d'attachement (Stovall \& Dozier, 2000, 2004). D'une part, des séquences vidéo ont été récoltées sur Internet, illustrant différents comportements parentaux. D'autre part, le journal d'attachement a été créé afin d'observer le processus de développement d'un nouvel attachement lorsque l'enfant arrive dans sa famille adoptive (Stovall \& Dozier, 2000, 2004). Le parent est invité à rapporter tous les soirs des situations où l'enfant a été en détresse (peur, douleur, séparation), impliquant donc son système d'attachement. Le parent y décrit le contexte et rapporte les comportements mis en place par l'enfant pour gérer cette situation. Le parent pratique de cette manière une observation fine des comportements de son enfant, tout en apprenant à décoder ceux-ci en fonction des repères apportés par la théorie de l'attachement. Ce type d'outil soutient le transfert du travail en séance vers les situations quotidiennes et permet de continuer à soutenir les habiletés d'observation, comme c'est le cas avec le vidéo-feedback.

En ce qui concerne l'engagement du parent (2), il s'agit d'un indicateur de la motivation pour une tâche d'apprentissage. Selon le modèle de Viau (2003), la motivation face à un apprentissage sera davantage soutenue si le parent perçoit que cette tâche lui permettra de progresser dans ses objectifs, s'il est suffisamment compétent pour la réaliser, s'il entretient une part active dans sa 
réalisation et s'il pourra en négocier une partie. C'est pourquoi le parent est invité à formuler des objectifs personnels dès la première séance, à les travailler à l'issue de chaque séance et à dégager les progrès réalisés. Une vigilance est apportée à la formulation des consignes de manière à ce que le parent se sente à la hauteur de ce qui est demandé.

En ce qui concerne la relation parent-intervenant (3), l'étude du VIPP (Stolk et al., 2008) a mis en évidence que l'alliance entre le parent et l'intervenant influence positivement l'évolution de la sensibilité parentale. Pour que le parent puisse fournir un caregiving sensible à son enfant, il doit se sentir en sécurité dans le cadre de l'intervention (Berlin, 2005 ; Schofield \& Beek, 2006). Changer ses comportements engendre une part de déséquilibre et de stress qui doit pouvoir être partagé avec l'intervenant. L'intervenant peut aussi, par son attitude soutenante, illustrer une certaine «façon de faire» offrant de la sécurité (Berlin et al., 2008). Se comporter de manière sécure pour l'intervenant revient à respecter le parent dans son style propre (Schofield \& Beek, 2006) et à s'adresser à lui en lui donnant un sentiment de compétence et non de menace (Juffer et al., 2008).

Au-delà de ces trois repères, les effets de l'apprentissage coopératif (Solar, 2001) semblent également pertinents pour notre intervention. En effet, les interactions suscitées par l'apprentissage coopératif incitent les participants à verbaliser et à reformuler leurs idées, à les confronter et à comparer leurs façons de faire. Parmi les interventions analysées, seul le COS s'organise en groupe tout en veillant à définir des objectifs spécifiques à chaque dyade parent-enfant (Marvin et al., 2002). Afin de permettre l'apprentissage coopératif, notre intervention sera basée sur cette méthodologie du COS. Une formule mixte sera mise en place dans notre intervention avec, d'une part, les première et dernière séances en individuel, consacrées à l'expression des motivations, la formulation d'objectifs spécifiques et à un retour sur ceux-ci en fin d'intervention et, d'autre part, les séances intermédiaires, organisées en groupe visant le partage d'expériences et les apports théoriques.

\subsection{Nos hypothèses}

Notre question de recherche porte sur l'efficacité de notre intervention, adressée à des parents adoptants belges francophones, visant à soutenir leur sensibilité parentale: cette intervention a-t-elle un effet sur la qualité de l'attachement de leur enfant adopté ?

Notre première hypothèse postule que les enfants adoptés améliorent leur attachement (plus de sécurité, moins de désorganisation) durant la période où leurs parents adoptants participent à l'intervention. L'arrivée dans la famille adoptive constitue un changement radical de contexte de soin et donc une occasion de remaniement de l'attachement pour l'enfant adopté (Stovall \& Dozier, 2004). Créés sur base d'expériences anglo-saxonnes, notre intervention a pour but de donner aux parents des clés de compréhension du vécu spécifique de leur enfant afin qu'ils répondent le plus adéquatement possible à leurs besoins d'attachement. Une évaluation de l'attachement des enfants, avant et après les ateliers, devrait mettre en évidence un attachement davantage sécure et moins désorganisé.

L'évaluation de la sensibilité parentale est difficile au-delà de la prime enfance car, à ce jour, il n'existe pas de mesures adéquates pour les enfants d'âge préscolaire. C'est pourquoi notre deuxième hypothèse porte sur le sentiment de compétence parentale comme étant un indicateur de cette sensibilité parentale. Ainsi, le sentiment de compétence du parent sera considéré comme ayant un effet sur l'attachement de l'enfant adopté. Dans un premier temps, il s'agit de mettre au clair si les parents adoptants participant à l'intervention améliorent leur sentiment de compétence parentale. Lors des contacts préliminaires avec les familles adoptives, de nombreux parents ont 
évoqué leur questionnement quant à leurs compétences à accompagner leur enfant. Ils expriment leur difficulté à éduquer un enfant se comportant différemment. En conséquence, les parents peuvent se sentir dépassés par les comportements rejetants, agressifs, ambivalents et contrôlants de leur enfant en détresse. Ils perdent alors confiance dans leur capacité à être des parents adéquats (Schofield \& Beek, 2006). Dès lors, notre intervention pourrait avoir comme effet d'amener les parents à être plus confiants dans leurs compétences parentales. Grâce à notre intervention, ils choisissent des repères éclairés pour l'éducation de leur enfant et se donnent les moyens de ne plus ressentir cette impuissance face à leur défi d'éducation (Schofield \& Beek, 2006). Sur base de cet apport, les parents pourraient se sentir davantage compétents à être une base de sécurité pour leur enfant, c'est-à-dire développer une relation nourrissante et affective, gérer ses comportements difficiles et placer des limites.

Dans un second temps, nous postulons que le sentiment de compétence parentale modère l'évolution de l'attachement de l'enfant. Plus l'évolution du sentiment de compétence parentale est élevée, la sécurité et la désorganisation de l'attachement de l'enfant s'améliorent. Notre intervention a pour but de permettre aux parents de se sentir davantage capables d'être une base de sécurité pour leur enfant. Un meilleur sentiment de compétence dans ce domaine pourrait les encourager à réagir davantage de manière sensible avec leur enfant, ce qui pourrait améliorer l'attachement de l'enfant.

\section{Méthodologie}

\section{1. Échantillon}

Deux canaux différents ont été utilisés pour le recrutement des familles. Un folder explicatif du projet a été posté sur des forums de discussion et envoyé aux neuf organismes d'adoption agréés (OAA) de la Communauté française de Belgique. De plus, la directrice de l'OAA « À la croisée des chemins » a contacté par téléphone quelques familles ayant adopté récemment un enfant. Au final, neuf familles ont participé au programme qui s'est déroulé d'avril à juin 2011.

L'échantillon comporte neuf enfants dont cinq garçons et quatre filles, ayant entre deux et 11 ans $(M=60$ mois, $E T=32,63)$. Huit enfants sur les neuf ont été adoptés après l'âge d'un an $(\mathrm{M}=36,6$ mois, $\mathrm{ET}=26,76)$. Bien que l'âge d'adoption joue un rôle dans l'adaptation de l'enfant (van den Dries et al., 2009), nous ne considérons pas cette variable dans nos analyses étant donné l'homogénéité de notre échantillon à cet égard. Tous les enfants de l'échantillon sont dans leur famille adoptive depuis au moins six mois $(M=23,11, E T=12,62)$. Parmi les neuf familles, les parents de cinq d'entre elles vivent en couple tandis que quatre sont des parents célibataires. Pour les couples, un papa n'a pas participé au programme et un autre a manqué les séances de groupe.

\subsection{Matériel expérimental}

\subsubsection{Mesure de l'attachement des enfants}

L'attachement de l'enfant, plus spécifiquement ses représentations, a été évalué en utilisant les «Histoires à compléter», version francophone de l'Attachment Story Completion Task (Bretherton, Ridgeway, \& Cassidy, 1990). Au cours de ce test, l'expérimentateur met en scène un début d'histoire représentant des situations de la vie quotidienne où un personnage enfant est placé dans des situations de détresse (maladresse accidentelle, transgression, peur, départ et retour des parents). Il est demandé à l'enfant de raconter la suite de l'histoire par l'utilisation de figurines. L'observation des réactions que l'enfant attribue aux personnages dans les différentes 
situations permet d'inférer ses attentes par rapport à la disponibilité et la présence sécurisante de ses figures d'attachement (Miljkovitch, Pierrehumbert, Karmaniola, \& Halfon, 2003).

Le narratif et les comportements de l'enfant sont codés à l'aide d'un tri de 65 cartes développé par Pierrehumbert et son équipe, les Cartes de codage pour les histoires à compléter $(\mathrm{CCH}$, Miljkovitch et al., 2003) décrivant les attitudes non-verbales et/ou le contenu et la cohérence du discours de l'enfant. Le codage se réalise en deux temps. D'abord, les cartes sont réparties en trois piles, allant du plus au moins caractéristiques de la prestation de l'enfant. Une seconde étape de «tri forcé » oblige à répartir les cartes en sept piles de «très caractéristique » à «très peu caractéristique », sur base d'un nombre prédéterminé de cartes par piles. Un score est attribué à chaque carte en fonction de la pile dans laquelle elle se trouve. Ainsi, il est possible d'évaluer le degré de ressemblance (scores de corrélation) des résultats de l'enfant avec les profils prototypiques d'enfants sécures et désorganisés, ceux-ci ayant établis par des experts de l'attachement.

Le narratif de l'enfant sécure montre qu'il envisage les aspects négatifs et positifs de l'histoire ainsi que les émotions vécues par les différents personnages. Il propose des situations constructives et réagit efficacement aux situations. Il peut appréhender des événements dangereux. L'enfant désorganisé, n'ayant pas de stratégie cohérente d'attachement, peut montrer des signes d'angoisse. Il rapporte des événements catastrophiques et son discours est peu cohérent (Miljkovitch et al., 2003).

Ce test a été présenté aux enfants lors des séances à domicile : au début de la première séance, en pré-test (T1), et en clôture de la dernière séance, en post-test (T2).

\subsubsection{Mesure du sentiment de compétence chez les parents}

L'Échelle globale du sentiment de compétence parentale (Meunier \& Roskam, 2009) est un questionnaire où le parent est invité à se positionner par rapport à 42 affirmations concernant la relation qu'il entretient avec son enfant. Considérant le sentiment de compétence parentale comme un concept multidimensionnel, les auteurs ont structuré le test autour de huit échelles. Dans notre analyse de résultats, deux échelles ont été utilisées, celles particulièrement en lien avec les questions traitées dans notre intervention :

- l'échelle «nurturance» évalue le sentiment du parent à pouvoir comprendre son enfant, lui montrer de l'affection et le réconforter;

- l'échelle «discipline» évalue le sentiment du parent à pouvoir être écouté de son enfant, le guider, gérer les limites et les émotions difficiles comme la colère.

Dans ce contexte, la notion de compétence parentale renvoie au sentiment qu'a le parent de fournir un caregiving adéquat à son enfant, tel que comprendre ses besoins d'attachement et y réagir de manière adéquate et cohérente, créer la confiance dans sa disponibilité et son accessibilité parentales, comprendre et accompagner l'enfant dans ses émotions difficiles et le guider en plaçant des limites. Les deux échelles semblent dès lors assez bien répondre à la notion de base de sécurité.

Comme pour l'évaluation de l'attachement de l'enfant, le sentiment de compétence du parent a été évalué en pré-test (T1) et en post-test (T2). Des analyses préliminaires indiquent qu'il n'y a pas de différence entre la mère et le père pour ces deux échelles au T1 (nurturance: $Z=-, 54$, $p=, 59$; discipline : $Z=-, 27, p=, 79$ ). 


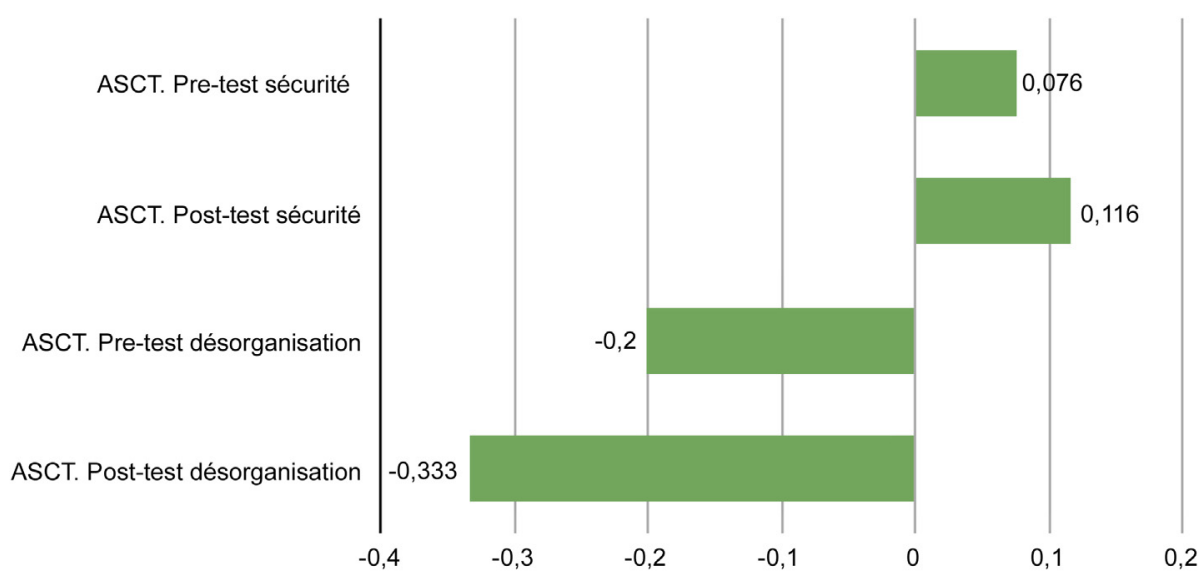

Fig. 1. Moyennes des résultats des enfants à l'ASCT en pré-test et en post-test.

\section{Résultats}

\section{1. Évolution de l'attachement chez les enfants}

En ce qui concerne la sécurité d'attachement, la moyenne des coefficients de corrélation est de,07 au T1 et de,12 au T2. Pour la désorganisation, la moyenne est de -,20 au T1 et -,33 au T2. Ces résultats indiquent que les scores moyens de la mesure d'attachement sont plus élevés au T2 qu'au T1 (Fig. 1).

Étant donné la taille réduite de notre échantillon, un test non paramétrique de Wilcoxon a été effectué. Celui-ci indique qu'il n'y a pas de différence significative entre les deux temps de mesure pour la sécurité $(Z=, 70, p=, 84)$ alors qu'une tendance apparaît pour la désorganisation $(Z=1,85, p=, 06)$.

\subsection{Influence du sentiment de compétence des parents sur l'évolution des représentations d'attachement de l'enfant}

Cette question a été travaillée en deux étapes. La première étape visait à comparer les moyennes des scores obtenus au T1 et au T2 pour les deux échelles «discipline» et «nurturance» afin de mettre en évidence une évolution, respectivement chez les mères et les pères (Fig. 2).

Aussi bien pour les mères que pour les pères, le score moyen pour l'échelle « discipline » semble s'améliorer du $\mathrm{T} 1$ (mère : 78,22; père : 77,80) au $\mathrm{T} 2$ (mère : 82,18; père : 82,40). Cependant, cette différence est non significative (mère: $Z=1,19, p=, 23$; père : $Z=, 94, p=, 34$ ). Pour l'échelle «nurturance », il semble que l'évolution ne soit pas identique chez les mères et les pères. Les scores moyens des mères ne présentent pas d'évolution entre le T1 $(20,22)$ et le T2 $(20,33)$ alors que les scores moyens des pères progressent entre le T1 $(18,60)$ et le T2 $(20,80)$. Cependant, comme pour l'échelle « discipline », aucune différence significative n'est observée pour les mères $(Z=-, 21, p=, 83)$ et les pères $(Z=-1,09, p=, 27)$.

Dans un second temps, les scores d'évolution du sentiment de compétence (différence entre les scores au T2 et au T1) des mères et des pères ont été corrélés au score d'évolution de l'attachement 


\section{COMPARAISON DES SCORES MOYENS POUR L'ÉCHELLE «DISCIPLINE»}

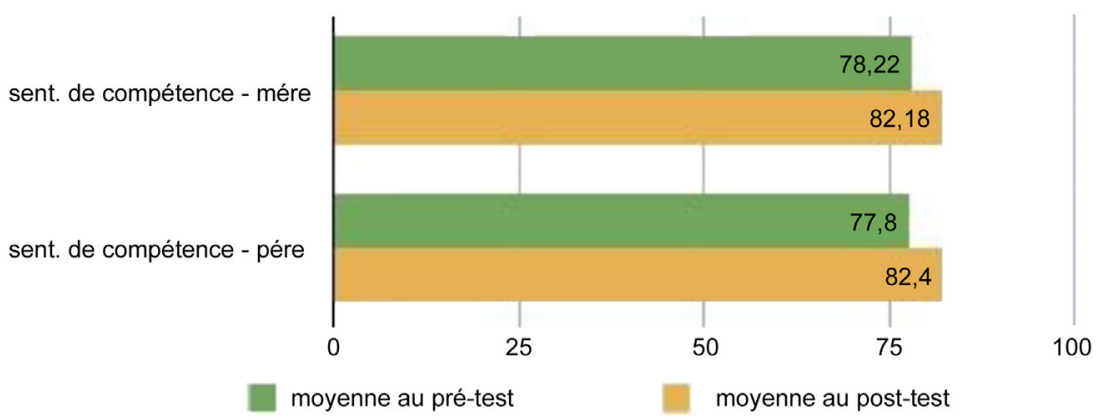

Fig. 2. Scores moyens du sentiment de compétence parentale au pré-test et au post-test chez la mère et le père pour l'échelle «discipline».

de l'enfant (différence entre les scores au T2 et au T1) pour voir si les deux évolutions sont liées Aucune corrélation n'est significative (Tableau 1).

Notons que la corrélation la plus haute est observée pour l'évolution du sentiment de compétence à l'échelle « discipline » entre la mère et le père $(r=, 50, p=, 39)$. Il semblerait que l'évolution de l'un influence positivement l'évolution de l'autre. Malgré la taille réduite de notre échantillon, les corrélations entre l'évolution de la désorganisation et l'évolution du sentiment de compétence aux deux échelles sont modérées. Cela suggère un attachement moins désorganisé chez l'enfant dont les parents améliorent leur sentiment de compétence sur ces deux échelles.

\section{Discussion}

L'objectif de cette étude vise à établir si une intervention brève centrée sur la sensibilité parentale, adressée à des parents adoptifs, améliore la qualité de l'attachement de leur enfant adopté. Dans cette démarche exploratoire, aucun résultat significatif n'a pu être dégagé même si des tendances se dessinent.

La première hypothèse, selon laquelle les enfants présentent un attachement davantage sécure et moins désorganisé à l'issue de l'intervention, a tendance à se confirmer. Après une intervention brève de quatre séances, la sécurité et principalement la désorganisation de l'attachement chez l'enfant semblent évoluer positivement. Ces résultats sont encourageants dans la mesure où la proportion d'enfants désorganisés est souvent sur-représentée dans les échantillons d'enfants adoptés (Juffer et al., 2005 ; Ponciano, 2010 ; van den Dries et al., 2009).

Tableau 1

Corrélations de Spearman entre l'évolution du sentiment de compétence des parents et l'évolution de l'attachement de l'enfant.

\begin{tabular}{|c|c|c|c|c|c|c|}
\hline & 1 & 2 & 3 & 4 & 5 & 6 \\
\hline 1. Discipline (mère) & - &, 50 &,- 14 &, 50 & ,09 & , 19 \\
\hline 2. Discipline (père) & & - &, 05 & ,00 &, 10 & ,30 \\
\hline 3. Nurturance (mère) & & & - &, 05 &, 13 &, 18 \\
\hline 4. Nurturance (père) & & & & - &, 10 &, 30 \\
\hline 5. Sécurité de l’attachement & & & & & - & , 19 \\
\hline 6. Désorganisation de l'attachement & & & & & & - \\
\hline
\end{tabular}


La seconde hypothèse cherche à affiner ces résultats en incluant le sentiment de compétence du parent comme modérant cette évolution. Si celui-ci se sent plus compétent pour être affectueux (échelle «nurturance») et pour donner de la structure à son enfant (échelle «discipline») à l'issue de l'intervention, l'évolution de l'attachement chez l'enfant pourrait en être influencé. Pour l'échelle «nurturance », le sentiment de compétence des mères ne semble pas évoluer alors que, chez les pères, une évolution semble apparaitre (non significative). Cette observation du côté des mères est assez étonnante puisque l'intervention, spécifiquement ciblée sur la sensibilité parentale, avait pour objectif de les aider à manifester de l'affection à l'égard de leur enfant. Lors du bilan qualitatif effectué à la fin de l'intervention avec chaque parent, deux mamans expliquent que, dès le début de l'intervention, elles se sentaient plutôt compétentes dans ce domaine. Cela pourrait expliquer l'absence d'évolution observée chez les mères. En revanche, dans le bilan qualitatif, trois pères mentionnent avoir été aidés à être plus complice avec leur enfant car ils comprennent mieux son comportement. Nous pouvons faire l'hypothèse que cette nouvelle complicité peut être une forme de nourriture affective.

En ce qui concerne l'échelle « discipline », tant les mères que les pères ont tendance à se sentir davantage compétents aux termes de l'intervention. Dans les commentaires du bilan qualitatif, certains parents se disent soulagés de percevoir plus concrètement les particularités de leur parentalité adoptive, les aidant ainsi à adapter leur système de caregiving aux besoins de leur enfant. Une explication de l'absence de différence significative pourrait être que les parents se sentant davantage conscients de la spécificité de la parentalité adoptive seraient plus exigeants envers eux-mêmes au terme de l'intervention, ce qui aurait pour effet de dissimuler l'évolution de leur sentiment de compétence. Allant dans ce sens, plusieurs parents ont fait la demande de séances complémentaires pour être accompagnés dans la mise en pratique des défis de leur parentalité adoptive dont ils ont pris conscience lors de l'intervention.

Par ailleurs, la corrélation moyenne (non significative) observée entre l'évolution du sentiment de compétence de la mère et du père pour l'échelle «nurturance » suggère l'importance de la coparentalité. Dès lors, il semble pertinent d'encourager le couple à participer ensemble aux séances d'intervention afin de renforcer leur évolution réciproque. Deux couples soulignent l'intérêt de discuter ensemble après les séances afin d'orienter leur démarche.

Pour répondre entièrement à notre question de recherche, notre dispositif souffre de quelques limites concernant l'échantillon, le dispositif lui-même et les variables prises en compte. Les différentes analyses statistiques ont conclu pour l'ensemble des hypothèses à des résultats non significatifs, probablement imputable à la taille réduite de l'échantillon. Les tendances observées mettent en évidence la pertinence de répliquer les analyses sur un échantillon plus large. De plus, les évolutions observées chez les enfants et les parents ne peuvent être attribuées avec certitude aux effets de l'intervention étant donné l'absence d'un groupe témoin. Nos résultats peuvent aussi être interprétés comme illustrant l'évolution spontanée de l'attachement de l'enfant et du sentiment de compétence des parents durant la période de l'intervention, sans que celle-ci ait joué un rôle spécifique dans cette évolution. Une autre remarque concerne le laps de temps entre les deux évaluations (six à huit semaines) : est-ce suffisant pour que des changements notoires soient observés? Il serait probablement utile de réaliser les mêmes évaluations à plus long terme afin de vérifier si cette évolution continue à opérer, ce qui pourrait se marquer avec plus de netteté dans les résultats observés.

Le choix des variables visant à évaluer l'efficacité de l'intervention peut également être questionné. L'évolution de l'attachement de l'enfant a été mesurée au niveau de ses représentations. N'aurait-il pas été plus réaliste d'évaluer des changements observés dans les comportements d'attachement de l'enfant comme étant des indices d'un premier effet de l'intervention ? Il est fort 
probable qu'une période où l'enfant teste de nouveaux comportements d'attachement précède les changements de ses représentations (Bowlby, 1969/1982). Par exemple, l'évaluation de l'IR (Moss et al., 2009) met en évidence des modifications de comportements de recherche de proximité comme précurseurs de changement de l'attachement chez l'enfant. De plus, l'enfant qui s'est forgé ultérieurement des représentations d'attachement, comme c'est le cas pour l'enfant adopté, ne développe pas directement un nouvel attachement mais sélectionne dans les interactions ce qui vient confirmer ses représentations déjà construites. Le changement se réalise progressivement grâce aux informations issues de ses interactions avec son nouveau parent, en expérimentant au jour le jour ses réactions et en testant petit à petit de nouveaux comportements d'attachement. Allant dans ce sens, plusieurs parents ont remarqué de nouveaux comportements chez leur enfant (par exemple: «il nous dit maintenant quand il a du mal », «il a eu le réflexe de m'appeler dans une situation de séparation qui était difficile», «il est plus posé quand il a une forte émotion et peut davantage m'en parler»).

Les effets encourageants obtenus à l'issue de l'intervention invitent à pointer les aspects qui devraient être maintenus dans une réplication de celle-ci. Les parents ont souligné le bon climat de travail. Considéré comme une clé de réussite (Berlin et al., 2008), une attention particulière y a été apportée, via de la convivialité, du respect et de la bienveillance. Ce climat a permis aux parents d'évoquer en toute confiance leur inquiétude et parfois leur impuissance, ce qui témoigne de l'expérience de la base de sécurité dans le cadre de l'intervention.

Cette brève intervention a également permis aux parents de prendre conscience de manière plus pointue des défis de leur parentalité adoptive. Il serait toutefois intéressant de comparer ces résultats avec ceux d'une intervention comprenant quelques séances complémentaires. Cela pourrait permettre aux parents d'échanger davantage et d'approfondir la mise en pratique des pistes leur permettant de répondre à leur enfant avec plus de sensibilité et de structure.

La formule mixte comprenant des temps en individuel et des temps en groupe offre les avantages des deux formules. D'une part, le temps individuel pour les parents a été consacré à un travail de définition des objectifs de l'intervention sur base des aspects primordiaux de la sensibilité parentale, à savoir le souci du parent de protéger et de soutenir l'enfant (Bigelow et al., 2010). D'autre part, le travail en groupe permet d'élargir le point de vue des parents à d'autres témoignages. Les participants se sont montrés favorables à cette formule mixte.

Enfin, les réactions des enfants lors des visites à domicile nous invitent à nous interroger sur l'interaction enfant-parent-intervenant dans le cadre de cette intervention. Un principe méthodologique consiste à ce que l'intervenant soit une base de sécurité pour le parent mais qu'en est-il pour l'enfant. Lors des visites à domicile, nous étions accueillis chaleureusement par les enfants qui collaboraient avec enthousiasme à l'évaluation. Certains enfants ont remercié leur parent de participer à cette intervention. Quelles sont les attentes de ces enfants à l'égard de cette intervention? Dans certains échanges, il nous semblait entendre tant chez les enfants que chez les parents la souffrance de ne pas pouvoir pleinement vivre leur relation d'attachement. Ainsi, les balises de la théorie de l'attachement explicitées dans le cadre de l'intervention recentrent les parents et l'enfant vers des attentes communes: la construction de la sécurité intérieure de l'enfant.

\section{Déclaration d'intérêts}

Les auteurs déclarent ne pas avoir de conflits d'intérêts en relation avec cet article. 


\section{Références}

Ainsworth, M. S., Blehar, M. C., Waters, E., \& Wall, S. (1978). Patterns of attachment: A psychological study of the strange situation. Oxford England: Lawrence Erlbaum.

Bakermans-Kranenburg, M. J., van Ijzendoorn, M. H., \& Juffer, F. (2008). Less is more: Meta-analytic arguments for the use of sensitivity-focused interventions. In F. Juffer, M. J. Bakermans-Kranenburg, \& M. H. van Ijzendoorn (Eds.), Promoting positive parenting: An attachment-based intervention (pp. 59-74). New York, NY: Taylor \& Francis Group/Lawrence Erlbaum Associates.

Berlin, L. J. (2005). Interventions to enhance early attachments: The state of the field today. New York, NY, US: Guilford Press.

Berlin, L. J., Zeanah, C. H., \& Lieberman, A. F. (2008). Prevention and intervention programs for supporting early attachment security. New York, NY, US: Guilford Press.

Bigelow, A. E., MacLean, K., Proctor, J., Myatt, T., Gillis, R., \& Power, M. (2010). Maternal sensitivity throughout infancy: Continuity and relation to attachment security. Infant Behavior \& Development, 33(1), 50-60. http://dx.doi.org/10.1016/j.infbeh.2009.10.009

Bowlby, J. (1969/1982). Attachment and loss: Vol I. Attachment. New York: Basic Books.

Bretherton, I., Ridgeway, D., \& Cassidy, J. (1990). Assessing internal working models of the attachment relationship: An attachment story completion task for 3-year-olds. Attachment in the preschool years: Theory, research and intervention. Chicago, IL: University of Chicago Press.

Coleman, P. K., \& Karraker, K. H. (1998). Self-efficacy and parenting quality: Findings and future applications. Developmental Review, 18(1), 47-85. http://dx.doi.org/10.1006/drev.1997.0448

Cyr, C., Euser, E. M., Bakermans-Kranenburg, M. J., \& Van Ijzendoorn, M. H. (2010). Attachment security and disorganization in maltreating and high-risk families: A series of meta-analyses. Development and Psychopathology, 22(1), 87-108. http://dx.doi.org/10.1017/s0954579409990289

De Wolff, M., \& van Ijzendoorn, M. (1997). Sensitivity and attachment: A meta-analysis on parental antecedents of infant attachment. Child Development, 68(4), 571-591. http://dx.doi.org/10.2307/1132107

Dozier, M., Higley, E., Albus, K. E., \& Nutter, A. (2002). Intervening with foster infants' caregivers: Targeting three critical needs. Infant Mental Health Journal, 23(5), 541-554. http://dx.doi.org/10.1002/imhj. 10032

Dozier, M., Lindhiem, O., \& Ackerman, J. P. (2005). Attachment and biobehavioral catch-up: An intervention targeting empirically identified needs of foster infants. New York, NY, US: Guilford Press.

Euillet, S., Spencer, R., Troupel-Cremel, O., Fresno, A., \& Zaouche-Gaudron, C. (2008). Les représentations d'attachement des enfants accueillis et des enfants adoptés. Enfance, 60(1), 63-70.

George, C., \& Solomon, J. (2008). The caregiving system: A behavioral systems approach to parenting. In J. Cassidy, \& P. R. Shaver (Eds.), Handbook of attachment: Theory, research, and clinical applications (2nd ed., pp. 833-856). New York, NY US: Guilford Press.

Howes, C., \& Spieker, S. (2008). Attachment relationships in the context of multiple caregivers. New York, NY, US: Guilford Press.

Jones, T. L., \& Prinz, R. J. (2005). Potential roles of parental self-efficacy in parent and child adjustment: A review. Clinical Psychology Review, 25, 341-363. http://dx.doi.org/10.1016/j.cpr.2004.12.004

Juffer, F., Bakermans-Kranenburg, M. J., \& van Ijzendoorn, M. H. (2005). The importance of parenting in the development of disorganized attachment: Evidence from a preventive intervention study in adoptive families. Journal of Child Psychology and Psychiatry, 46(3), 263-274. http://dx.doi.org/10.1111/j.1469-7610.2004.00353.x

Juffer, F., Bakermans-Kranenburg, M., \& Van Ijzendoorn, M. (2008). Promoting positive parenting an attachment based-intervention. New-York: Laurence Erlbaum.

Juffer, F., \& Rosenboom, L. G. (1997). Infant-mother attachment of internationally adopted children in the Netherlands. International Journal of Behavioral Development, 20(1), 93-107. http://dx.doi.org/10.1080/016502597385469

Marcovitch, S., Goldberg, S., Gold, A., \& Washington, J. (1997). Determinants of behavioural problems in Romanian children adopted in Ontario. International Journal of Behavioral Development, 20(1), 17-31. http://dx.doi.org/10.1080/016502597385397

Marvin, R., Cooper, G., Hoffman, K., \& Powell, B. (2002). The Circle of Security project: Attachmentbased intervention with caregiver-pre-school child dyads. Attachment \& Human Development, 4(1), $107-124$. http://dx.doi.org/10.1080/14616730252982491

Meins, E., Fernyhough, C., Fradley, E., \& Tuckey, M. (2001). Rethinking maternal sensitivity: Mothers' comments on infants' mental processes predict security of attachment at 12 months. Journal of Child Psychology and Psychiatry, 42(5), 637-648. http://dx.doi.org/10.1111/1469-7610.00759 
Meunier, J. C., \& Roskam, I. (2009). Self-efficacy beliefs amongst parents of young children: Validation of a self-report measure. Journal of Child and Family Studies, 18(5), 495-511. http://dx.doi.org/10.1007/s10826-008-9252-8

Miljkovitch, R., Pierrehumbert, B., Karmaniola, A., \& Halfon, O. (2003). Les représentations d'attachement du jeune enfant. Développement d'un système de codage pour les histoires à compléter. Devenir, 15(2), $143-177$. http://dx.doi.org/10.3917/dev.032.0143

Moss, E., Dubois-Comtois, K., Cyr, C., Carignan, M., \& St-André, M. (2009). Évaluation d'un programme d'intervention visant à optimiser la sécurité affective et le développement moteur et cognitif des enfants placés en famille d'accueil. Montréal: Conseil canadien sur l'apprentissage.

O’Conner, T. G., Marvin, R. S., Rutter, M., Olrick, J. T., \& Britner, P. A. (2003). Child-parent attachment following early institutional deprivation. Development and Psychopathology, 15(1), 19-38. http://dx.doi.org/10.1017/s0954579403000026

Ponciano, L. (2010). Attachment in foster care: The role of maternal sensitivity, adoption, and foster mother experience. Child \& Adolescent Social Work Journal, 27(2), 97-114. http://dx.doi.org/10.1007/s10560-010-0192-y

Schofield, G., \& Beek, M. (2006). Handbook for foster care and adoption. London: British association for adoption and fostering.

Slade, A. (2005). Parental reflective functioning: An introduction. Attachment and Human Development, 7(3), $269-281$. http://dx.doi.org/10.1080/14616730500245906

Solar, C. (2001). Le groupe en formation des adultes : Comprendre pour mieux agir. Bruxelles: De Boeck.

Stolk, M. N., Mesman, J., van Zeijl, J., Alink, L. R. A., Bakermans-Kranenburg, M. J., \& van Ijzendoorn, M. H. (2008). Early parenting intervention aimed at maternal sensitivity and discipline: A process evaluation. Journal of Community Psychology, 36(6), 780-797. http://dx.doi.org/10.1002/jcop.20280

Stovall, K. C., \& Dozier, M. (2000). The development of attachment in new relationships: Single subject analyses for 10 foster infants. Development and Psychopathology, 12(2), 133-156. http://dx.doi.org/10.1017/s0954579400002029

Stovall, K. C., \& Dozier, M. (2004). Forming attachments in foster care: Infant attachment behaviors during the first 2 months of placement. Development and Psychopathology, 16(2), 253-271.

Tyrrell, C., \& Dozier, M. (1999). Foster parents' understanding of children's problematic attachment strategies: The need for therapeutic responsiveness. Adoption Quarterly, 2(4), 49-64. http://dx.doi.org/10.1300/J145v02n04_04

van den Dries, L., Juffer, F., van Ijzendoorn, M. H., \& Bakermans-Kranenburg, M. J. (2009). Fostering security? A meta-analysis of attachment in adopted children. Children and Youth Services Review, 31(3), 410-421. http://dx.doi.org/10.1016/j.childyouth.2008.09.008

van Londen, W. M., Juffer, F., \& van Ijzendoorn, M. H. (2007). Attachment, cognitive, and motor development in adopted children: Short-term outcomes after international adoption. Journal of Pediatric Psychology, 32(10), 1249-1258. http://dx.doi.org/10.1093/jpepsy/jsm062

Viau, R. (2003). La motivation en contexte scolaire ( $3^{\mathrm{e}}$ ed., pp. ). Bruxelles: De Boeck.

Weinfield, N. S., Whaley, G. J. L., \& Egeland, B. (2004). Continuity, discontinuity, and coherence in attachment from infancy to late adolescence: Sequelae of organization and disorganization. Attachment \& Human Development, 6(1), 73-97. http://dx.doi.org/10.1080/14616730310001659566 